\title{
A Transplant Website in Today's World
}

\author{
Jacob A. Akoh* and Peter Caton
}

\begin{abstract}
Directorate of Surgery \& Renal Services, Plymouth Hospitals NHS Trust, Derriford Hospital, Plymouth PL6 8DH, United Kingdom
\end{abstract}

\begin{abstract}
With the aid of a professional developer, the South West Transplant Centre designed and launched a website in September 2006 to provide patient friendly information in all aspects of renal transplantation and health professionals with transplant related e-book protocols alongside research materials, printable referral forms and statistical data including survival figures. The aim of this paper is to report on our experience of the first year of operating such a website. The website consists of approximately 500 pages covering subjects ranging from transplant related procedures to the transplant procedure itself. During the first year the total number of hits on SWTC website were 70,971 with April 2007 having a peak of 10,357 hits. There was sustained interest in the website throughout the year and the amount of information (kilobytes) downloaded was highest during the first three months. Regular visitors to the website come from several countries across the globe including the United Kingdom, New Zealand, Seychelles, Canada, France and the US. A well-designed website is a useful tool for increasing awareness of Unit activities, increasing organ donation, providing information and in some instances training people in geographically remote areas. To maintain relevance transplant websites require to be upgraded on a regular basis by transplant clinicians and relevant sections expanded to meet the needs of the public.
\end{abstract}

\section{INTRODUCTION}

Recent advances in information technology have produced the internet revolution. There is a wealth of information on the world wide web and given appropriate search engines, access to virtually any type of information at all times, almost anywhere in the world is possible. In view of the potential benefits of operating a website, the South West Transplant Centre (SWTC) set up a committee to study the feasibility, design and launch of a website in September 2005. It was felt that a website would increase the profile of the SWTC and increase awareness for patients and health professionals about transplantation, particularly about organ donation; provide a ready and suitable resource to other units particularly in developing countries who perform transplantation or are about to set up a transplant unit; and provide clear guidelines and protocols for managing various conditions relating to transplantation. The aim of this paper is to report our experience of the first year of operating such a website.

\section{DESIGN}

Before the development of the website, the SWTC had no online presence. The time from the initial decision to develop a website for the SWTC to launch was 12 months. The website committee raised funds (approximately $£ 10,000$ ) to support the development of a transplant website and hired the services of a professional developer to design and oversee its implementation. The targeted audience was primarily patients, family and friends, physicians, the press, hospital

*Address correspondence to this author at the Directorate of Surgery \& Renal Services, Level 03, Derriford Hospital, Plymouth PL6 8DH, United Kingdom; Tel: 01752 763619; Fax: 01752 774651;

E-mail: Jacob.akoh@phnt.swest.nhs.uk staff and the public. The developer carried out research by talking to a representative sample of the above and made recommendations to the website committee. A transplantbased website (http://www.swtransplant.com) was launched in September 2006, created and designed by Open Brand and Marketing and constructed by PL9 Design. The sitemap includes a Home Page, Patient, Carer, Donor, Clinicians, About Us pages and useful links to other sites.

The website consists of approximately 500 pages covering transplant related subjects. It also provides health professionals with transplant related e-book protocols alongside research materials, printable referral forms and transplant related statistical data including survival figures. For patients or visitors to the SWTC, the website offers a directions service. Entering a post code in the appropriate page automatically links to Google maps, providing a detailed map and direction to the SWTC. There are also pages containing information about where to stay in Plymouth for visitors to the SWTC. New and exciting developments within the transplant centre are also published on the site.

The SWTC is working towards complying with Priority 1 and where possible Priority 2 guidelines of the World Wide Web Consortium's (W3C) Web Content Accessibility Guidelines [1]. The website has been designed with the following accessibility guidelines in mind:

- The site uses a style sheet and relative font sizes on all text with the exception of graphical text. The font size of text can be increased or decreased in size by using "view" option in the browser

- All images, where appropriate, have an alternative text attribute - when an image is conveying important information its content is described with an alternative text

- No information is exclusively conveyed using colour. 
This website has been developed to be viewable Internet Explorer 6.0+ and Mozilla Firefox 1.0+ on both Windows and Macintosh platforms.

A "hit" is a request to the web server for a file, image or HTML (HyperTextMarkupLanguage) page whereas a "visit" refers to access to the web site. One visit may generate many hits.

\section{FINDINGS}

During the first year the total number of hits on SWTC website was 70,971 with April 2007 having a peak number of 10,357 hits. Fig. (1) shows that there was sustained interest in the website throughout the year. The amount of information (kilobytes) downloaded was highest during the first three months. An analysis of regular visitors to the site is shown in Table 1. Table $\mathbf{1}$ also compares interest in the website during the first and twelfth months of the first year. During the first month of the website, there were visitors from the United States, United Kingdom, Seychelles and nonprofit organisations (names not displayed on feedback) whereas twelve months later, people from across Europe, South America and North Africa visited the website.

Table 2 shows the pages most commonly accessed. There was widespread interest in the website with the "about" section and "patient" pages generating more interest.

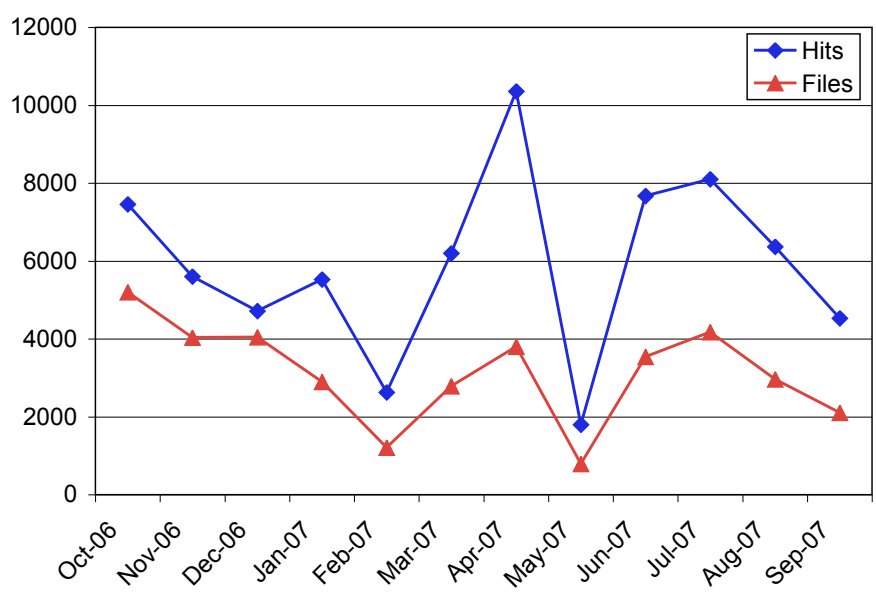

Fig. (1). SWTC Website - Hits and files accessed during the first year (October 2006 to September 2007).

\section{DISCUSSION}

In addition to general information about the SWTC, the website captures the patient's journey from imminent renal failure to transplantation and beyond. A transplant website is a useful resource to people with diverse interests ranging from health professionals to patients or interested public. It has a wealth of transplant educational material and informa-

Table 1. South West Transplant Centre Website Usage, Sample at Months 1 and 12

\begin{tabular}{|c|c|c|c|c|}
\hline US Commercial & $3017(40.64)$ & 2209 (42.49) & $1798(39.65)$ & $1325(36.24)$ \\
\hline Unknown & 749 (10.04) & $529(10.17)$ & $958(21.12)$ & $863(23.61)$ \\
\hline Seychelles & $24(0.32)$ & $12(0.23)$ & $24(0.53)$ & 120.33 ) \\
\hline Italy & 0 & 0 & $71(1.57)$ & $68(1.86)$ \\
\hline Australia & 0 & 0 & $20(0.44)$ & $20(0.55)$ \\
\hline Colombia & 0 & 0 & $4(0.09)$ & $4(0.11)$ \\
\hline Morocco & 0 & 0 & $3(0.07)$ & $1(0.03)$ \\
\hline Germany & 0 & 0 & $2(0.04)$ & $2(0.05)$ \\
\hline Denmark & 0 & 0 & $2(0.04)$ & $1(0.03)$ \\
\hline Brazil & 0 & 0 & $1(0.02)$ & $1(0.03)$ \\
\hline Canada & 0 & 0 & $1(0.02)$ & $1(0.03)$ \\
\hline Poland & 0 & 0 & $1(0.02)$ & $1(0.03)$ \\
\hline
\end{tabular}

*Website accessed from Domain name.net.

Figures in parentheses are percentages. 
tion and it is no surprise that people from both the developed and developing nations across the world visit the SWTC website on a regular basis. Considering the young age of the website, the number of hits (Fig. 1) are high and it is reasonable to assume that the website has fulfilled most of the aspirations of the Unit during the first year. It is not clear why the peak number of hits was in April 2007. A significant reason behind the success of this site is the well (professionally) designed website reflecting the total patient experience. Publishing results of the SWTC activity and providing useful links to other transplant related information have also contributed to this success. Our experience demonstrates that the internet provides a level playing field in offering equal opportunities to all to sell their ideas or products no matter how small or large the organisation. The internet is also an effective medium for highlighting unit activities and results. For a centre such as the SWTC serving a number of renal units, the website offers a unique opportunity to both patients and health professionals to access current information and protocols.

Given the interest generated in the website around the world, it is probable that the website has helped to promote the SWTC both nationally and internationally. While it is difficult to determine how much benefit a visitor derives from the website, it is reasonable to assume that the continued increase in the number of visits from various countries around the globe suggest that some need is being met. People from the Seychelles have visited our website on a regular basis since its inception. Good quality educational material for health providers including transplant multidisciplinary teams is limited in both quantity and quality. With improving access to the internet, even in developing countries, medical websites may play a major role in the delivery of up to date educational material [2]. However, following a detailed assessment of 94 websites on kidney transplantation, Hanif et al. [3] concluded that the educational material was of generally poor quality. More input from transplant clinicians and regular updates of materials on such sites are required.

Table 2 shows the top 10 groups of pages visited on the website during September 2007. After the homepage, the most popular was the 'about' section that describes the hospitals and the staff involved in providing transplant care in the South West Transplant Centre. The next popular groups of pages visited including 'patient', 'clinicians', 'donor' and 'carer' indicate the overall interest in core information regarding the patients journey - probably accessed by patients and their close family seeking information about the transplant procedure, what it entails and the risks involved. Also the site provides a source of information to clinicians about the protocols in effect in the SWTC, the statistics and information about immunosuppression and complications. The lack of analysed feedback is a major drawback of this study. Feedback is the life force of any website and measures to rectify this are being taken.

It is thought that the most visited websites were often not ranked among the best sites to provide information [4]. By creating dedicated patient pages that take into consideration the diverse reading levels, effective information dissemination would be ensured. The UK recently altered the regulations governing organ donation to allow altruistic nondirected living kidney donation. The donor section of the SWTC website can be expanded to provide clear information about the process of non-directed donation or paired exchange. The United Network for Organ Sharing uses a Transplant Living website to provide information for potential live kidney donors and to counter the effects of solicitation for organs in the US [5]. Perhaps the SWTC website by providing a similar service will increase the number of potential kidney donors. The demand for immediate and accurate information will increase in all sectors of human experience, particularly so in the health sector. Our experience will serve as a catalyst for other transplant centres in the UK and around the world.

In today's world, a website can be used to educate and inform both the general public and healthcare professionals around the globe. Our experience proves that a well-designed website is a useful tool for increasing awareness of the SWTC activities, increasing organ donation and providing information to people especially to those in remote parts. To continue to be relevant transplant websites require to be up-

Table 2. Top Ten Pages of the Website Visited During September 2007

\begin{tabular}{|c|c|c|c|c|}
\hline \multicolumn{2}{|c|}{ Hits } & \multicolumn{2}{c|}{ Uisits } & Home page \\
\hline \hline 194 & $4.28 \%$ & 143 & $22.99 \%$ & $\underline{\text { /about/ }}$ \\
\hline 346 & $7.63 \%$ & 107 & $17.20 \%$ & /patient/ \\
\hline 304 & $6.70 \%$ & 92 & $14.79 \%$ & /clinicians/ \\
\hline 329 & $7.25 \%$ & 72 & $11.58 \%$ & /donor/ \\
\hline 236 & $5.20 \%$ & 61 & $9.81 \%$ & /carer/ \\
\hline 152 & $3.35 \%$ & 46 & $2.40 \%$ & /conditions.php \\
\hline 23 & $0.51 \%$ & 14 & $2.09 \%$ & /accessibility.php \\
\hline 26 & $0.42 \%$ & 13 & $2.09 \%$ & /privacy.php \\
\hline
\end{tabular}


graded on a regular basis by transplant clinicians and relevant sections expanded to meet the needs of the public.

\section{ACKNOWLEDGEMENTS}

We wish to acknowledge the significant role played by members of the Website Committee - Dr. Peter Rowe, Consultant Nephrologist, Mr. Jamie Barwell, Consultant Vascular and Transplant Surgeon, Wendy Colwell, Renal Computer Systems Manager and Sharon Bradshaw, Transplant Co-ordinator and Peter Caton, Transplant Support Practitioner.

\section{REFERENCES}

[1] Web Content Accessibility Guidelines. http://www.w3.org/TR/ WCAG10/

[2] Stevenson C. Educational resources: website and book review. Anaesthesia 2007; 62(Supl 1): 103-7.

[3] Hanif F, Abayasekara K, Willcocks L, et al. The quality of information about kidney transplantation on the World Wide Web. Clin Transplant 2007; 21: 371-6.

[4] Moody EM, Clemens KK, Storsley L, Waterman A, Parikh CR, Garg AX. Donor nephrectomy outcomes research (Donor) network. Improving on-line information for potential kidney donors. Kidney Int 2007; 71: 1062-70.

[5] Delmonico FL, Graham WK. Direction of the organ procurement and transplantation network and united network for organ sharing regarding the oversight of live donor transplantation and solicitation for organs. Am J Transplant 2006; 6: 37-40.

(C) Akoh and Caton; Licensee Bentham Open.

This is an open access article licensed under the terms of the Creative Commons Attribution Non-Commercial License (http://creativecommons.org/licenses/ by-nc/3.0/) which permits unrestricted, non-commercial use, distribution and reproduction in any medium, provided the work is properly cited. 